\title{
Data Submission Model
}

National Cancer Institute

\section{Source}

National Cancer Institute. Data Submission Model. NCI Thesaurus. Code C142496.

Standards for information provided to authorizing officials for marketing purposes. 